\title{
Asociación entre confianza e influencia negativa con el rendimiento académico como desencadenante de la deserción escolar en la educación media superior
}

\author{
Association Between Confidence and Negative Influence with Academic \\ Performance as a Trigger for School Dropout in High School
}

\section{Associação entre confiança e influência negativa com o desempenho acadêmico como gatilho para o abandono escolar no ensino médio}

David Fernando Lozano Treviño

Universidad Autónoma de Nuevo León, Nuevo León, México davidflozano@gmail.com http://orcid.org/0000-0002-5455-4223

Lauro Maldonado Maldonado Universidad Autónoma de Nuevo León, Nuevo León, México lamaldon@hotmail.com http://orcid.org/0000-0002-5071-0348

\section{Resumen}

La deserción en la educación media superior es uno de los retos más grandes en materia escolar que tienen las autoridades mexicanas. Analizar los factores que la causan es de sumo interés tanto para comprender el fenómeno como para proponer estrategias y líneas de acción para abatirla.

El objetivo de la presente investigación fue señalar la asociación que existe entre los constructos de confianza e influencia negativa con el rendimiento académico que lleva a la deserción escolar en la educación media superior. El estudio fue no experimental, descriptivo y correlacional, con una muestra de 2881 desertores en los 32 estados de México, los cuales 


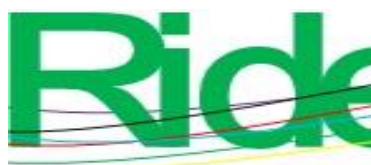

Revista Iberoamericana para la

Investigación y el Desarrollo Educativo

ISSN $2007-7467$

se agruparon por regiones. Se corrió una regresión lineal múltiple con los factores independientes Confianza e Influencia negativa, y Rendimiento académico como factor dependiente. Se propuso un modelo teórico que refleja las variables comprendidas en los factores. Los resultados mostraron que no existe asociación entre aquellos dos con este último, el rendimiento académico que lleva a la deserción escolar en el nivel medio superior, por lo que no se planteó un modelo empírico.

Lo cierto es que la confianza que un alumno tenga en sus autoridades escolares, profesores, padres de familia y amigos, así como la influencia que estos ejerzan en los primeros, tiende a beneficiar sus asistencias, promedios y aprobaciones, pero son otros factores los que sólidamente se asocian con el rendimiento académico que lleva a la permanencia o deserción escolar en el nivel educativo ya mencionado.

Palabras clave: confianza, deserción escolar, educación media superior, influencia negativa, rendimiento académico.

\section{Abstract}

Desertion in high school is one of the biggest challenges in school matters that Mexican authorities have. Analyzing the factors that causes it is of great interest both to understand the phenomenon and to propose strategies and lines of action to reduce it.

The purpose of this investigation was to point out the association between the constructs of confidence and negative influence on academic performance that leads to high school dropout. The study was non-experimental, descriptive and correlational, with a sample of 2881 deserters representing the 32 Mexican states. A multiple linear regression was run with the independent factors Confidence and Negative influence, and Academic performance as a dependent factor. A theoretical model that reflects the variables included in the factors was proposed. The results showed that there is no association of confidence and negative influence with academic performance that leads to high school dropout, so an empirical model wasn't proposed.

The truth is that the confidence that a student has in their school authorities, teachers, parents and friends, as well as the influence they exercise in the former, tends to benefit their attendance, averages and approvals, but other factors are those that solidly are associated with the academic performance that leads to the permanence or drop-out in high school. 


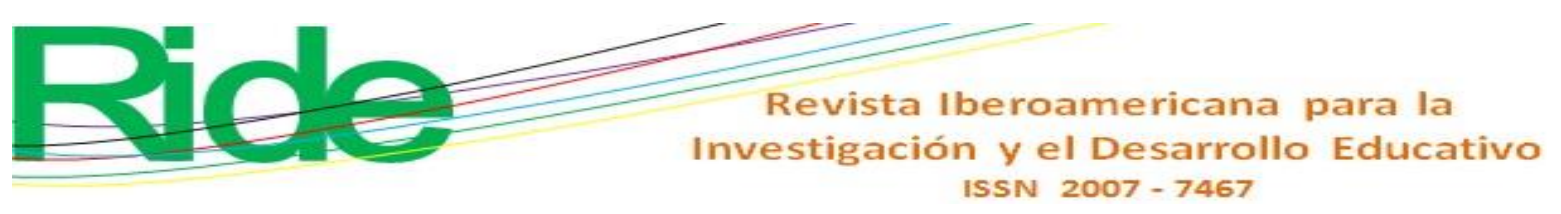

Keywords: confidence, school dropout, high school education, negative influence, academic performance.

\section{Resumo}

O atrito no ensino médio é um dos maiores desafios em matéria escolar que as autoridades mexicanas enfrentam. Analisando os fatores que causam, é de grande interesse entender o fenômeno e propor estratégias e linhas de ação para derrubá-lo.

O objetivo da presente investigação foi apontar a associação existente entre os construtos de confiança e influência negativa com o desempenho acadêmico que leva ao abandono escolar no ensino médio. O estudo foi não experimental, descritivo e correlacional, com uma amostra de 2881 desertores nos 32 estados do México, agrupados por região. Uma regressão linear múltipla foi realizada com os fatores independentes Confiança e Influência Negativa e Desempenho Acadêmico como fator dependente. Foi proposto um modelo teórico que reflete as variáveis incluídas nos fatores. Os resultados mostraram que não há associação entre os dois com o último, o desempenho acadêmico que leva ao abandono escolar no ensino médio, portanto, não foi levantado um modelo empírico.

A verdade é que a confiança que um aluno tem nas autoridades escolares, professores, pais e amigos, bem como a influência que exercem no primeiro, tende a beneficiar sua frequência, médias e aprovações, mas outros fatores são aqueles que Eles estão solidamente associados ao desempenho acadêmico que leva à permanência ou abandono escolar no nível educacional acima mencionado.

Palavras-chave: confiança, evasão, ensino médio, influência negativa, desempenho acadêmico.

Fecha Recepción: Mayo 2019

Fecha Aceptación: Octubre 2019 


\section{Introducción}

Para erradicar la deserción escolar en la educación media superior (EMS) es necesario ir más allá de penurias económicas o falta de becas; para su comprensión es menester acercarse a los jóvenes, ponerse en sus zapatos, entender sus contextos y situaciones, así como su desarrollo biológico y psicológico (Juárez, 2018). Es un reto complicado de no fácil solución, pues una respuesta no necesariamente impacta positivamente en otro factor generador de abandono escolar (Consejería de Educación de la Junta de Castilla y León [CE], 2013; Román, 2013). Se trata, pues, de una de las problemáticas sociales y educativas de mayor envergadura, ya que determina el éxito de los planes y programas en materia educativa, además de que se asocia con la injusticia social (Romero y Hernández, 2019); provoca inequidad, marginación social (Díaz y Osuna 2017; Venegas, Chilusia, Castro y Casillas, 2017) y vulnerabilidad laboral (Landeros, 2012). Sin duda, su erradicación es una de las principales competencias del sistema educativo (Roca, 2009); y el desenlace del fracaso escolar (Venegas et al., 2017).

La deserción de un alumno se deriva de rasgos de pasividad, desorganización, ansiedad y baja autoestima que a su vez provocarán una tendencia a devaluar al individuo que abandonó (Lara, González, González y Martínez, 2014). Se genera escasez de oportunidades y pérdida de recursos gubernamentales, pues lo asignado a la educación del estudiante con miras a incrementar su calidad de vida se pierde. La planeación y coordinación educativa debe direccionarse a propiciar la permanencia escolar hasta el término de los estudios (Moreno, 2013). Para Romero y Hernández (2019), las metas en materia del éxito académico focalizados a incrementar los niveles de educación obligatoria son ambiciosas: al aumentar los años de estudio, se incrementa el peligro de exclusión del sistema escolar.

Infante y Parra (2010) aseguran que la deserción escolar es una barrera para el desarrollo. Si la población no está cubierta por el sistema educativo, difícilmente generarán el desarrollo necesario para enfrentar la desigualdad y erradicar la pobreza.

Ciertamente, en la gran mayoría de los casos, la deserción escolar se da por voluntad propia debido a que el estudiante no logra involucrarse lo suficiente con el ambiente intelectual y social de la escuela (Miranda, 2018). El ausentismo y la reprobación son aspectos altamente mencionados en los estudios científicos (Cuellar, 2017; Arroyo, Montes, Reyes, Zamudio y Tapia, 2017; Ruiz, García

y Pérez, 2014; Martínez, Hernández, Carrillo, Romualdo y Hernández, 2013; Vidales, 2009; Ponce, 2004). Lo anterior debido a que los alumnos que reprueban alguna materia tiene 2.47 veces más 


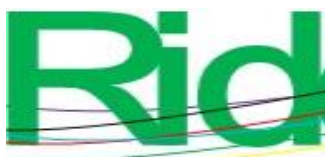

Revista Iberoamericana para la

Investigación y el Desarrollo Educativo

ISSN $2007-7467$

probabilidades de abandonar la escuela con respecto a los que no reprueban (Secretaría de Educación Pública [SEP], 2015). López, Velázquez e Ibarra (2011) afirman precisamente que lo que desencadena la deserción escolar es la reprobación.

Sin lugar a duda, la deserción escolar es el resultado de la acumulación y combinación de diversos aspectos que comprenden al propio individuo, su familia, amigos, la comunidad, el sector productivo y el propio sistema educativo. La acumulación y el lamentable vigor con el que se da el desenganche, la desvinculación, el alejamiento, la retirada y el ausentismo desenlazan en la deserción escolar (Salvà, Oliver y Comas, 2014); los cuales se dan diariamente en el actuar escolar desde la educación básica y se manifiestan en la EMS, cuyos factores, al parecer, se fortalecen entre ellos y en donde todos los que componen una sociedad son culpables (Mena, Fernández y Riviére, 2010; Espínola y Claro, 2010; Martínez, 2009; Díaz y Osuna, 2017).

El objetivo principal de la educación como sistema es que los estudiantes se certifiquen mediante la adquisición de conocimientos y habilidades científicos, tecnológicos y sociales, y promover actitudes y valores positivos (Poy, 2010); busca impulsar la flexibilidad e innovación en los procesos de enseñanza, que la educación entre en una dinámica de perpetuidad y autoaprendizaje, que los graduados coadyuven al desarrollo sustentable del país y que sean capaces de adentrarse en contextos nacionales e internacionales (El Sahili, 2011). Una realidad es que para muchos estudiantes que abandonan la EMS lo anterior no tiene ningún significado, pero esto adquiere sentido en función del siguiente nivel (Poy, 2010), es decir: la educación superior, la vida laboral y el ejercicio de la ciudadanía.

Por lo anterior, y ya que concluir la EMS provoca beneficios en materia económica, fiscal, laboral, salud y educativa (Camacho, 2018), es fundamental asegurar que un mayor número de jóvenes permanezcan en la escuela, concluyan y tengan oportunidad de cursar tramos superiores.

Si bien son muchos los factores que se asocian con la deserción escolar, como ya se mencionó líneas atrás, en el presente estudio nos enfocaremos en la reprobación, los promedios en calificaciones y el ausentismo como variables desencadenantes, que reflejan una potencial deserción de los estudiantes y que pudieran estar correlacionadas con factores como la confianza que los alumnos tienen en directores, maestros, orientadores vocacionales, familiares y amigos, así como la manera en la que estos influyen negativamente en los primeros.

Por lo anterior, la pregunta de investigación es la siguiente: ¿De qué manera se asocia la confianza de los alumnos hacia directores, maestros, familiares y amigos, así como la influencia negativa de estos en el rendimiento académico como principal determinante de la deserción escolar? 


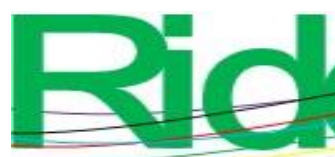

Por lo tanto, el objetivo es asociar los constructos conceptualizados como Confianza e Influencia negativa con el factor Rendimiento académico como principal determinante de la deserción escolar en EMS.

\section{Factores asociados con la deserción escolar en la EMS}

En términos generales, la deserción escolar es la interrupción o el abandono de los estudios. Sin embargo, la complejidad que encierra como objeto de investigación requiere un análisis más profundo en su conceptualización. Así, puede ser entendida como el alejamiento del medio escolar y de la formación académica por parte de los estudiantes provocado por el propio individuo, su familia, su entorno comunitario, la economía o desconfianza en el propio centro educativo (Ortega, Macías y Hernández, 2014; Gómez y Vázquez, 2014); es desertar las tareas y los tiempos dedicados al procesos de enseñanza-aprendizaje en un instituto (Moreno, 2013; Abril, Román, Cubillas y Moreno, 2008); es la apatía del alumnado por la instrucción colegial motivado por dificultades sensitivas, culturales y sociales (El Sahili, 2011); es el proceso en el que los alumnos se desligan de cualquier elemento que conforma el sistema educativo (Silvera, 2016); se manifiesta de manera rápida y acumulativa distanciándose de la escuela (Salvà et al., 2014); son todas aquellas personas que se encuentran entre las edades de 18 a 24 años y que no han concluido la EMS, o bien que al término de la secundaria no continuaron sus estudios de bachillerato (CE, 2013).

Existen dos tipos de deserción escolar: 1) intracurricular, que se revela en el transcurso del semestre, y 2) intercurricular, que se da entre el fin del ciclo y el comienzo del próximo (Consejo para la Evaluación de la Educación del Tipo Medio Superior [Copeems], 2012). Según Landeros (2012), la primera comprende $57.2 \%$, al tiempo de que la mayoría de los alumnos que llegan al final del tercer semestre o ciclo, según sea el caso, logran finalizar su preparación media superior. Para Abril et al. (2008) al concluir los estudios básicos y durante los dos primeros semestres del bachillerato es que se decide desertar de la EMS.

Múltiples son los constructos que causan el abandono. En el presente estudio nos enfocaremos en aquellos que se relacionen con los factores aquí estudiados: familiares, pedagógicos, cognitivos, escolares y personales. Además, la adolescencia juega un papel importante, pues el estudiante experimenta transformaciones físicas y cambios psicológicos en el curso: deja de ser un niño para convertirse en adulto (Ortega et al., 2014). A continuación, se detallan los factores mencionados: 


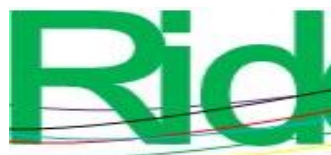

a) Familiares: Incluye el compromiso familiar con los estudios, los valores practicados, el equilibrio familiar y la adscripción laboral temprana (Ortega et al., 2014); la unión familiar, contraer roles de adulto, padres permisivos y de escasa supervisión, bajas metas familiares (Salvà et al., 2014); los grados académicos de los padres (Alegre y Pérez, 2010); hermanos que hayan abandonado niveles educativos básicos o de media superior, madres jóvenes (Mora, 2010); copiosos integrantes en la familia, falta de papá o mamá, padres sin empleo (Casquero y Navarro, 2010); padres indígenas, la posición que se ocupa entre los hermanos (Antelm, Gil y Cacheiro, 2015); provenir de etnias (Landeros, 2012); sobreproteccionismo o sobreexigencia parental (El Sahili, 2011); familias disfuncionales (Camacho, 2018); el involucramiento de los padres con la institución educativa, los amigos de sus hijos, el grado de confianza e influencia que hay entre los miembros de la familia (Copeems, 2012).

b) Pedagógicos: Los procesos de revisión y calificación, la manera en la que se organizan las actividades escolares, relación docente-alumno, excesiva disciplina por parte de los profesores (Ortega et al., 2014); no cubrir los requerimientos de los alumnos, no ahilar los estudios de bachillerato con el plan de vida que tenga el alumno (Moreno, 2013); influencias y actitudes inapropiadas de los profesores, aulas sin control, bajo acompañamiento, carente actualización del profesorado (Salvà et al., 2014); poca coordinación y trabajo colegiado entre docentes, deficiente tutorización, o bien que esta no se da de forma personalizada, débil convivencia escolar, entornos inseguros, acoso sexual y ascetismo (CE, 2013); no contar con trabajadores sociales ni psicólogos, nulas charlas sobre proyectos de vida, habilidades socioemocionales y valores (Gómez y Vázquez, 2014); pocas esperanzas de los docentes hacia sus alumnos (Roca, 2009; Mirete, Soro y Maquilón, 2015); la reprobación, promedios bajos, no presentarse a clases, alumnos desinteresados en sus propias clases, dividir alumnos con promedios aceptables de aquellos con promedios bajos (Mena et al., 2010); las prácticas de estudio (Poy, 2010); los resultados obtenidos en matemáticas, la cantidad de actividades extraescolares (Mora, 2010); alumnos con extraedad (Casquero y Navarro, 2010); el grado de dificultad de las unidades de aprendizaje (El Sahili, 2011); materias acreditadas de manera extraordinaria (Díaz y Osuna, 2017); poco interés en la investigación, bajas habilidades de comunicación (Gómez y Vázquez, 2014); exámenes uniformados, así como la influencia y la confianza que los maestros generen en los alumnos (Copeems, 2012).

c) Cognitivos: Deficiente rendimiento académico (Mora, 2010); trayectos académicos marcados por malogrados aprendizajes, desconocimiento del idioma en el que se imparten las clases 


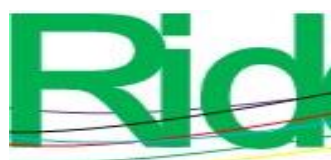

Revista Iberoamericana para la

Investigación y el Desarrollo Educativo

ISSN $2007-7467$

(Roca, 2009); déficits de atención y concentración por parte del estudiante (Ortega et al., 2014), y no recibir apoyo instruccional según las necesidades personales del alumno (Antelm et al., 2015).

d) Escolares: Directores líderes, las cualidades de la enseñanza y de los instrumentos educativos (Antelm et al., 2015); la fama de la institución académica (El Sahili, 2011); deficiencias en la orquestación que hay entre niveles básicos y el medio superior, la relación que haya entre la academia y el sector productivo y social, incluyendo la familia (Gómez y Vázquez, 2014); la modalidad de la preparatoria y la confianza hacia el director, los maestros, el orientador y el prefecto (Copeems, 2012).

e) Personales: Género (Casquero y Navarro, 2010); embarazos adolescentes, hacerse cargo de hermanos más pequeños, desinterés para asistir a la escuela (Infante y Parra, 2010); mala alimentación, falta de adaptación al nuevo nivel educativo y entorno social; inmadurez intelectual, insuficientes habilidades y conocimientos (Gómez y Vázquez, 2014); bajas metas académicas, delincuencia, adicciones, influencias negativas, desconexión de las relaciones con los compañeros, falta de referentes positivos, amigos con bajos grados académicos, pensar que el estudio es una pérdida de tiempo, disgusto con el estudio o la escuela, desmotivación ante los malos resultados en las materias (Salvà et al., 2014); bajas habilidades socioemocionales (CE, 2013); relacionar el estudio con el aburrimiento, pensar que dejar los estudios hace positivamente diferente a una persona, pretender atraer la atención de padres, docentes o amigos (Mena et al., 2010); el compromiso con los estudios (Antelm et al., 2015); sentirse frustrado ante malos resultados, no relacionar el estudio con la mejora en la calidad de vida (El Sahili, 2011); los alicientes individuales, la afectividad (Martínez, 2009); fumar, el uso que se le da al tiempo de ocio, la participación en clase, las influencias de la pareja y los amigos (Copeems, 2012).

\section{El rendimiento académico y la deserción escolar en la EMS}

De acuerdo con la SEP (2014a), los tres factores que más aumentan la deserción escolar se relacionan con el ámbito escolar: baja asistencia, reprobación y promedios bajos, es decir, el rendimiento académico.

El rendimiento académico es la consecuencia de lo aprendido según la valoración realizada por el profesor mediante exámenes, tareas, trabajos grupales y otras actividades adicionales como la asistencia a clases (Saucedo, Herrera, Díaz, Bautista y Salinas, 2014). Se refiere a los estudiantes que 


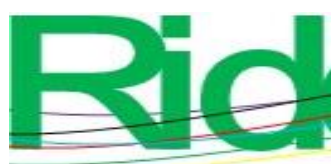

Revista Iberoamericana para la

Investigación y el Desarrollo Educativo

ISSN $2007-7467$

logran la certificación de las competencias educativas del nivel cursado, esto es, que concluyen satisfactoriamente sus estudios (Antelm et al., 2015).

Sin duda, el factor de mayor peso en la deserción escolar es el bajo rendimiento académico (López et al., 2011). Se trata de uno de los retos más desafiantes en México, ya que aquellos que fracasan desertan sin los conocimientos ni habilidades necesarios para desarrollarse en la vida. Cabe señalar que el bajo rendimiento se evidencia con la reprobación, el ausentismo y los promedios bajos (Vidales, 2009; Cuellar, 2017; Copeems, 2012).

Abandonar los estudios y no asistir a clases están asociados, puesto que esta última es comienzo de la primera (Camacho, 2018). Aquellos estudiantes que reprueban materias, tienen bajos promedios y que frecuentemente se ausentan de clases son los más proclives a desertar de la escuela (Silvera, 2016). Los bajos resultados son el desencadenante de una apostasía causada por diversas variables (Salvà et al., 2014), tal y como la reprobación (Landeros, 2012; Juárez, 2018), a la que le anteceden acciones como el ausentismo y los bajos promedios académicos (Díaz y Osuna, 2017; Martínez, 2009).

Ciertamente, diversos estudios han relacionado el rendimiento académico con la deserción escolar. En un análisis hecho al programa del Movimiento Contra el Abandono en México se ubicó que reprobar materias es la segunda causa para desertar la EMS, con $23 \%$, así como que $74 \%$ de los que abandonaron reprobaron materias en el semestre anterior (SEP, 2015). Por su parte, López et al. (2011) ubicaron que todos los alumnos que desertaron sus estudios reprobaron alguna vez una materia, mientras que $65.6 \%$ no se presentaba con sus tutores. Similar fue lo que Tapia, Tamez y Tovar (1994) informaron: $62.6 \%$ de los estudiantes desertores habían reprobado una o más materias. Mientras que Ruiz et al. (2014) indicaron que el principal factor asociado con la deserción escolar era la reprobación de materias: 31.4\% de los estudiantes abandonaron la escuela porque no aprobaron alguna, $22.9 \%$ lo hicieron por desinterés en los estudios, lo que provocaba ausentismo, y $11.4 \%$ por no lograr promedios aprobatorios. Arroyo et al. (2017) registraron que $32.7 \%$ de los desertores mostraron reprobación en el ciclo escolar anterior, mientras que el bajo rendimiento académico se daba, entre otros motivos, por no acudir a clases o no dedicar suficiente tiempo al estudio. En su mayoría, los alumnos que no aprueban lo hacen por promedios mediocres, faltan a sus clases o destinan poco tiempo a estudiar (Vidales, 2009). En esa misma tónica, Díaz y Osuna (2017) informaron que $89 \%$ de los desertores reprobaron una materia en el último ciclo escolar que cursaron. Finalmente, la SEP (2014) registró que no acudir a clases en todo un día o a cinco asignaturas en una semana es causa de riesgo de deserción, al igual que no acreditar más de una unidad de aprendizaje. 


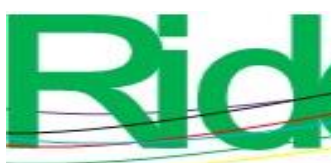

Revista Iberoamericana para la

Investigación y el Desarrollo Educativo

ISSN $2007-7467$

Así, para el presente estudio el rendimiento académico será el resultado obtenido al final del curso conformado principalmente por la aprobación, o en su caso reprobación, los promedios obtenidos y la asistencia a clases.

\section{La confianza hacia directivos, docentes, familiares y amigos en alumnos desertores de EMS y las influencias negativas}

Así como la comprensión y el agrado por el trabajo escolar son sentimientos positivos por los que atraviesan los estudiantes, la confianza hacia los directores, maestros, familiares y amigos también lo es (Artavia, 2005; Vera y Mazadiego, 2010). Su estímulo se da cuando se les invita a expresar sugerencias, se fomenta el diálogo, se privilegia la sana convivencia escolar y el sentido de pertenencia tanto de los estudiantes como de sus familias con la escuela. La confianza se genera debido a que el profesor sabe motivarla con el contacto individual y las recomendaciones que da a sus estudiantes (Diario Oficial de la Federación [DOF], 2008).

Sin duda, las buenas relaciones entre docentes y alumnos que generan confianza y comunicación en ambos sentidos inciden en el rendimiento académico (Ministerio de Educación, Cultura y Deporte [MECD], 2012). Por lo que se puede afirmar que los docentes que inspiran confianza cuentan con habilidades socioafectivas (Campos, Brenes y Solano, 2010); saben entender a los demás, propician buenas relaciones y saben ser amigos (Fernández, 2013); se ocupan de la comunidad estudiantil (Piña, Escalante, Ibarra y Fonseca, 2017); son abiertos y empáticos, accesibles, respetan distintas formas de pensar, inspiran confianza, saben llamar la atención y son sensibles a los intereses y requerimientos de los alumnos simplemente con el contacto que tienen con ellos (Covarrubias y Piña, 2004; Moreno, Sandoval y Valdés, 2015).

El rendimiento académico también está relacionado con la atención que brindan los padres y el ambiente familiar del estudiante (Sánchez, Téllez, Sánchez y Reyes, 2017). El compromiso y participación de la familia, así como lo es el del profesor, son importantes. Los desertores abandonan sus estudios sin haber recibido algún tipo de apoyo de padres, hermanos, profesores ni directivos escolares (Silvera, 2016); o bien, si estos lo hicieron, fue insuficiente, puesto que las relaciones entre ellos son poco significativas (Salvà et al., 2014). En ese sentido, la convivencia familiar y escolar favorecen el rendimiento académico y, por lo tanto, la continuidad de los estudios (SEPa, 2014). El primer factor identificado con el pobre rendimiento académico es el propio desinterés de la familia (CE, 2012); se agrega también la falta de comunicación y supervisión (Díaz y Osuna, 2017; Camacho, 


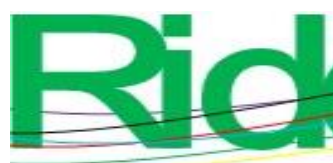

Revista Iberoamericana para la

Investigación y el Desarrollo Educativo

ISSN $2007-7467$

2018); la escasa interacción familiar (Martínez, 2009); problemas con la familia y el nivel de acercamiento entre maestros y alumnos (Abril et al., 2008; Dzay y Narváez, 2012); las insuficientes prácticas de estudio entre padres e hijos y la actitud negativa de la familia hacia las actividades académicas. A su vez, se menciona el poco involucramiento del director de la escuela (Antelm et al., 2015); las prácticas negativas de los padres hacia temas escolares de sus hijos, la nula confianza entre ellos y hacia los maestros (Copeems, 2012); la deficiente convivencia entre docentes y alumnos, así como la desconfianza hacia la administración de la escuela (Landeros, 2012; Román, 2013). Al respecto, Reyes, Trejo y Topete (2017) ubicaron en su estudio que ser confiable es una de las características fundamentales que debería tener un director de escuela.

Además, Ortega et al. (2014) reportaron que $66 \%$ de los docentes consideran que para que los jóvenes no deserten es necesario tanto generar confianza entre maestros y alumnos como hablar con los padres de familia para que hagan lo mismo. Puesto que, siguiendo esta vez a Mora (2010), a mayor deficiencia en la relación con los padres, mayor predisposición a abandonar. En el trabajo de Espínola y Claro (2010), por ejemplo, aquellos alumnos cuyos padres se involucraron en sus tareas escolares presentaron poco ausentismo, mejores promedios, mayor aprobación y se sintieron más motivados con sus estudios. Los alumnos prefieren docentes confiables para cursar sus unidades de aprendizaje (Sánchez y Gaya, 2014; Rossado, 2016). Según datos de la SEP (2014), 79.5 \% de los desertores no son buscados por algún docente o directivo, mientras que los padres encuestados afirmaron que si hubieran generado confianza con sus hijos brindándoles apoyo y ejecutando actividades en conjunto la deserción escolar no se hubiera dado.

Por lo anterior, confianza se entiende como la seguridad que experimenta un estudiante de EMS para acudir con directivos, profesores, orientadores, familiares y amigos y expresar opiniones y problemas relativos al rendimiento escolar.

Por otro lado, Miranda (2018) destacó que la mayoría de las deserciones manifestadas por la reprobación, promedios bajos y ausentismo son voluntarios, y se originan porque el alumno no logró integrarse al entorno de estudio ni social de la institución educativa. La SEP (2008), mientras tanto, ubicó que el rendimiento académico deficiente se da porque los estudiantes acceden a la EMS con insuficientes conocimientos, la desigualdad social, el ambiente escolar y considerar que obtendrán poco provecho por seguir estudiando. Tapia et al. (1994) agregan las insuficientes herramientas y conocimientos que utilizan los docentes en sus clases, además de que identificaron que $99 \%$ de los estudiantes consideraron que si ellos mismos se lo propusieran, aumentarían su rendimiento académico. En dichos estudios, la confianza no fue mencionada; a los hábitos y técnicas de estudio, 


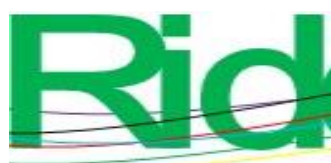

Revista Iberoamericana para la

Investigación y el Desarrollo Educativo

ISSN $2007-7467$

por el contrario, se les da un peso importante. En esa línea, Arroyo et al. (2017) y Vidales (2009) señalan que el bajo rendimiento académico se da porque los alumnos no toman apuntes en clase y dedican poco tiempo al estudio.

Fonseca (2016), Barrales, Gómez y Guerrero (2015), Mirete et al. (2015), Saucedo et al. (2014), Martínez et al. (2013) y Abril et al. (2008) enlistan factores personales como la frustración, baja autoestima, depresión, desinterés, insuficiencia de conocimientos o habilidades, expectativas desalentadoras, la comprensión de asignaturas y tener flojera como causantes de bajos rendimientos académicos.

Las influencias que reciben los alumnos de EMS son un factor que se asocia al rendimiento escolar y por lo tanto a la deserción.

Como se ha visto, la familia es capaz de influir en la vida de cualquiera (Díaz y Osuna, 2017). Ortega et al. (2014) señalan que $100 \%$ de los estudiantes desertores pretenden retomar sus estudios si cuentan con el apoyo de sus padres. Dentro de las influencias que manifiestan los familiares hacia los alumnos y que merman o benefician el rendimiento académico y llevan o alejan a la deserción se encuentran los problemas con alguno de los miembros (Infante y Parra, 2010); exigua connivencia de la familia en la escuela, miembros que hayan abandonado el bachillerato (Salvá et al., 2014; Mora, 2010); el oficio de los papás y el estilo familiar para valorar los estudios y las actitudes hacia el trabajo escolar y la propia institución educativa (Romero y Hernández, 2019; Antelm et al., 2015); las proyecciones futuras que los padres hacen de sus hijos (Roca, 2009); el bajo nivel académico de los padres (Alegre y Pérez, 2010; Copeems, 2012); patrones educativos inadecuados al interior de la familia y falta de control de los padres (Camacho, 2018); la participación que hay entre padres e hijos en la elaboración de actividades escolares en el hogar, el estudio, el tiempo destinado a las tareas, la disciplina, asistencia y acompañamiento a la escuela y a las clases, el interés por las calificaciones, la construcción de ideas positivas sobre la educación y el estímulo a la práctica del arte y el deporte. Los padres que influyen asertivamente en sus hijos inciden en mejores promedios, mayor asistencia a clases e incrementan las probabilidades de que éstos concluyan sus estudios (SEP, 2014b).

Ahora bien, Moreno (2013) ubicó que, en el ámbito escolar, dentro de las influencias negativas que recibía un estudiante y que afectaban su rendimiento académico, se encontraban prácticas didácticas desmotivadoras y actitudes perniciosas de los docentes. El comportamiento apático del profesor, no atender cuestionamientos u opiniones de los alumnos, ser intolerante, impartir clases excesivamente técnicas, no destinar tiempo a dinámicas de preguntas y respuestas y no motivar a sus estudiantes son comportamientos que alejen a los alumnos del interés académico (García, García y 


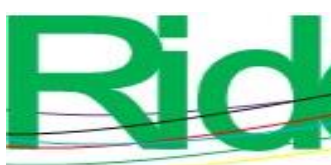

Revista Iberoamericana para la

Investigación y el Desarrollo Educativo

ISSN $2007-7467$

Reyes, 2014). Aunado a directivos relajados y a prácticas como agrupar los salones de clases según las calificaciones obtenidas por los alumnos (Romero y Hernández, 2019; Antelm et al., 2015).

Dentro de las influencias positivas se pueden mencionar las expectativas que los profesores tienen de sus alumnos, el completo uso del tiempo de clases, la calidad de la enseñanza que ejercen los maestros (Roca, 2009); docentes que son buenos referentes para sus estudiantes (Mirete et al., 2015); profesores que celebran la dedicación y entrega al estudio, así como la mejora en promedios, aprobación de materias y la asistencia a clases, dan retroalimentación y elogian el empeño puesto (MECD, 2012); asesoran individualmente a los alumnos, los motivan y hacen públicos sus logros educativos (Campos et al., 2010; Oviedo y Oviedo, 2017); brindan acompañamiento (Miranda, 2018); seguimiento y apoyo (DOF, 2008); la activación de los profesores ante situaciones conflictivas (Covarrubias y Piña, 2004); la intensidad con la que los maestros se involucran en la orientación académica (Torres, Badillo, Valentín y Ramírez, 2014); se mantienen cercanos a los adolescentes (Moreno et al., 2015); aclaran dudas en clase (Kopyto, 2017). También influyen los directivos que se involucran con el estudiante y su familia, principalmente con aquellos que tienen un rendimiento académico deficiente, que ejercen control en las clases, apoyan propuestas estudiantiles, programan e involucran a los jóvenes en actividades extraescolares, supervisan los recesos y planean y ejecutan programas de orientación vocacional y tutorías (SEP, 2014b).

En materia de las influencias de amistades se pueden mencionar los propios consejos y acciones negativas, el destino que se le da al tiempo libre con los amigos (Díaz y Osuna, 2017); influjos de grupos o pandillas (Camacho, 2018); no ser capaz de relacionarse con los compañeros o con su propio entorno social, poca convivencia con referentes positivos, amistades sin estudios o que hayan abandonado el bachillerato, el poco valor que los amigos le dan a la academia (Salvá et al., 2014; Landeros, 2012; Alegre y Pérez, 2010); amistades vándalas, alcohólicas y con poco interés por la cultura (Romero y Hernández, 2019); que conducen a vicios y actos delictivos (Ruiz et al., 2014); las proyecciones que las amistades tienen de su propio futuro (Roca, 2009); escasas amistades de grados más avanzados que funjan como alguna especie de tutores, y el hecho de contar con amigos irrespetuosos (SEP, 2014a).

Por lo tanto, influencia negativa se entiende como aquellas acciones, estilos de vida, atenciones o consejos de directivos, docentes, familiares y amigos que guían la toma de decisiones de los alumnos de EMS.

Sin embargo, a pesar de que Sánchez et al. (2017) y Oviedo y Oviedo (2017) relacionaron la atención de los familiares y docentes con el rendimiento académico, le dieron mayor peso a factores 
motivacionales, a las estrategias de aprendizaje de los estudiantes, así como a los estilos de enseñanza de los profesores, la generación de ambientes que facilitan el desarrollo integral, el autoaprendizaje colaborativo y el desarrollo de la propia enseñanza. Además, el rendimiento académico positivo se da por la dedicación que se tenga en el estudio, la reflexión de lo aprendido y la praxis de los contenidos (Chimborazo y Zoller, 2018); la claridad en la evaluación del docente (Lemus et al., 2015); el diseño de las actividades (Alcántara, 2009); la organización y sucesión de los contenidos, la forma en la que se explican y motivan los cuestionamientos en los propios estudiantes (Barragán et al. 2010); la capacidad de llamar la atención de los alumnos y retenerla (Cuéllar, 2017), y la actualización de los temas que se ven en clase (Organista, 2010).

Por último, Reyes et al. (2017) manifestaron que más que influir en los estudiantes, los directivos deben velar por la perpetuidad del aprendizaje.

\section{Método}

El presente estudio es no experimental, descriptivo y correlacional. Se utilizó la base de datos de la Encuesta Nacional de Deserción en la Educación Media Superior (Endems) aplicada por el Copeems (2012). La recolección de datos fue mediante entrevistas personales en vivienda con un cuestionario estructurado. La muestra se conformó por 2881 desertores de la EMS en los 32 estados agrupados por regiones.

La construcción del modelo teórico se hizo con base en dos factores independientes, a saber, Confianza e Influencia negativa, y uno dependiente, Rendimiento académico. Las variables medibles y las preguntas aplicadas se aprecian en la tabla 1.

El primero constructo explicativo independiente, Confianza, fue conformado por la seguridad que experimenta un estudiante de EMS para recurrir a las siguientes personas cuando enfrenta problemas en la escuela: $a$ ) director o subdirector, $b$ ) algún maestro, $c$ ) a un orientador o prefecto, $d$ ) a su papá, e) a su mamá,f) a algún amigo y $g$ ) a sus hermanos. Se obtuvo un nivel de fiabilidad, por medio del alfa de Cronbach $(\alpha)$, de 0.732 , lo que se considera como aceptable (George y Mallery, 2003), por lo que las variables miden un mismo constructo y están relacionadas (Welch y Comer, 1988).

Para el segundo, Influencia negativa, las variables fueron los influjos que recibieron los alumnos para que dejaran de estudiar por parte de las siguientes personas: $a$ ) la mamá, $b$ ) el papá, $c$ ) otros familiares, $d$ ) la pareja, $e$ ) amigos y $f$ ) alguien más. La fiabilidad expresada fue buena, con 0.899. 


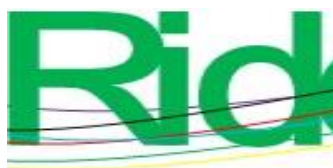

Revista Iberoamericana para la

Investigación y el Desarrollo Educativo

ISSN $2007-7467$

En cuanto al constructo dependiente, Rendimiento académico, las variables consideradas fueron: $a$ ) la asistencia a clases, $b$ ) la reprobación y $c$ ) el promedio. La fiabilidad obtenida fue de 0.689, algo débil pero cercana a lo aceptable.

Tabla 1. Modelo teórico

\begin{tabular}{|c|c|c|}
\hline Constructo & Variable & Pregunta en la Endems \\
\hline \multirow{7}{*}{ Confianza } & $\mathrm{C} 1$ & $\begin{array}{l}\text { Cuando tenías problemas en la escuela, ¿qué tanta } \\
\text { confianza tenías para recurrir al director o } \\
\text { subdirector? }\end{array}$ \\
\hline & $\mathrm{C} 2$ & $\begin{array}{l}\text { Cuando tenías problemas en la escuela, ¿qué tanta } \\
\text { confianza tenías para recurrir a algún maestro? }\end{array}$ \\
\hline & $\mathrm{C} 3$ & $\begin{array}{l}\text { Cuando tenías problemas en la escuela, ¿qué tanta } \\
\text { confianza tenías para recurrir a un orientador o } \\
\text { prefecto? }\end{array}$ \\
\hline & $\mathrm{C} 4$ & $\begin{array}{l}\text { Cuando tenías problemas en la escuela, ¿qué tanta } \\
\text { confianza tenías para recurrir a tu papá? }\end{array}$ \\
\hline & $\mathrm{C} 5$ & $\begin{array}{l}\text { Cuando tenías problemas en la escuela, ¿qué tanta } \\
\text { confianza tenías para recurrir a tu mamá? }\end{array}$ \\
\hline & C6 & $\begin{array}{l}\text { Cuando tenías problemas en la escuela, ¿qué tanta } \\
\text { confianza tenías para recurrir a algún amigo? }\end{array}$ \\
\hline & $\mathrm{C} 7$ & $\begin{array}{l}\text { Cuando tenías problemas en la escuela, ¿qué tanta } \\
\text { confianza tenías para recurrir a tus hermanos? }\end{array}$ \\
\hline \multirow{6}{*}{ Influencia negativa } & IN1 & $\begin{array}{l}\text { ¿Tu mamá influyó en la decisión de que dejaras de } \\
\text { estudiar? }\end{array}$ \\
\hline & IN2 & $\begin{array}{l}\text { ¿Tu papá influyó en la decisión de que dejaras de } \\
\text { estudiar? }\end{array}$ \\
\hline & IN3 & $\begin{array}{l}\text { ¿Otros familiares influyeron en la decisión de que } \\
\text { dejaras de estudiar? }\end{array}$ \\
\hline & IN4 & $\begin{array}{l}\text { ¿Tu pareja influyó en la decisión de que dejaras de } \\
\text { estudiar? }\end{array}$ \\
\hline & IN5 & $\begin{array}{l}\text { ¿Tus amigos influyeron en la decisión de que } \\
\text { dejaras de estudiar? }\end{array}$ \\
\hline & IN6 & $\begin{array}{l}\text { ¿Alguien más influyó en la decisión de que dejaras } \\
\text { de estudiar? }\end{array}$ \\
\hline \multirow{3}{*}{ Rendimiento académico } & RA1 & Asistencia a clases durante el último año de estudio \\
\hline & RA2 & $\begin{array}{l}\text { Materias reprobadas durante los estudios de } \\
\text { bachillerato }\end{array}$ \\
\hline & RA3 & $\begin{array}{l}\begin{array}{l}\text { Promedio del último año de estudios del } \\
\text { bachillerato }\end{array} \\
\end{array}$ \\
\hline
\end{tabular}

Fuente: Elaboración propia con base en el Copeems (2012)

\section{Resultados}

Una vez obtenidos los promedios de las variables para conformar los constructos, se procedió con los estadísticos descriptivos. 
Tabla 2. Estadísticos descriptivos

\begin{tabular}{|l|l|l|l|}
\hline & Media & Desv. & $\mathbf{N}$ \\
\hline Confianza & 2.4774 & 0.71575 & 2549 \\
\hline $\begin{array}{l}\text { Influencia } \\
\text { negativa }\end{array}$ & 3.7379 & 0.40230 & 2549 \\
\hline $\begin{array}{l}\text { Rendimiento } \\
\text { académico }\end{array}$ & 2.3010 & 0.64340 & 2549 \\
\hline
\end{tabular}

Fuente: Elaboración propia con base en el Copeems (2012)

Como se aprecia en la tabla 2, la media del constructo dependiente es de 2.3010, es decir, los alumnos desertores tenían en promedio un rendimiento superior a "algo" o "regular", pues a veces faltaban a clases, alguna vez reprobaron alguna materia o el promedio era regular, pero no era uno de los mejores. La confianza hacia directores, maestros, orientadores, padres, amigos o hermanos fue de 2.4774, es decir, entre "algo" y "poco". Para Influencia negativa se obtuvo una media de 3.7379, es decir, los padres, familiares, parejas o amigos influyeron entre "poco" y "nada" en la decisión de que los alumnos abandonaran sus estudios de EMS. En referencia a esta última variable, con el objeto de hacer comparaciones entre variables que miden con escala de cuatro, se ajustó la escala empleada, multiplicándola por cuatro y sustrayéndole dos (Muñiz et al., 2011).

En seguida, se obtuvieron las correlaciones entre variables, tal y como se muestra en la tabla 3. Resalta la correlación significativa en el nivel 0.01 que hay entre Confianza y Rendimiento académico con 0.136 .

Tabla 3. Correlaciones

\begin{tabular}{|l|l|l|l|}
\hline & Confianza & $\begin{array}{l}\text { Influencia } \\
\text { negativa }\end{array}$ & $\begin{array}{l}\text { Rendimiento } \\
\text { académico }\end{array}$ \\
\hline Confianza & 1.000 & 0.018 & 0.136 \\
\hline $\begin{array}{l}\text { Influencia } \\
\text { negativa }\end{array}$ & 0.018 & 1.000 & -0.031 \\
\hline $\begin{array}{l}\text { Rendimiento } \\
\text { académico }\end{array}$ & 0.136 & -0.031 & 1.000 \\
\hline
\end{tabular}

Fuente: Elaboración propia con base en el Instituto Nacional para la Evaluación de la Educación [INEE] (2017) 


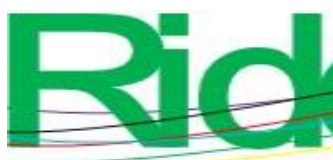

\section{Revista Iberoamericana para la Investigación y el Desarrollo Educativo \\ ISSN $2007-7467$}

Posteriormente, para comprobar las relaciones entre los constructos expuestos en el modelo teórico, se aplicó una técnica de regresión lineal múltiple representada con la siguiente ecuación:

$$
y=\beta 1+\beta 1 x 1+\beta 2 x 2+e
$$

Donde:

$\mathrm{X} 1$ = Confianza;

$\mathrm{X} 2$ = Influencia negativa;

$y=$ Rendimiento académico.

En la tabla 4 se aprecia un coeficiente de determinación $\mathrm{R}^{2}$ de 0.020 , es decir, el grado en el que el modelo explica el comportamiento de la variable dependiente con respecto a las independientes, o bien la relación que hay de las $x$ con la $y$. Con el valor arrojado, se determina que la relación entre Confianza e Influencia negativa con Rendimiento académico es muy pequeña o prácticamente no existe, por lo que se acepta la no influencia de los constructos regresores en el factor dependiente. Por lo que, además, no se genera un modelo empírico.

Tabla 4. Estadísticas de regresión para la ecuación 1.

\begin{tabular}{|l|l|}
\hline \multicolumn{2}{|l|}{ Estadísticas de la regresión } \\
\hline Coeficiente de correlación múltiple & 0.140 \\
\hline Coeficiente de determinación $\mathrm{R}^{\wedge} 2$ & 0.020 \\
\hline Error típico & 0.63727 \\
\hline Durbin-Watson & 1.814 \\
\hline
\end{tabular}

Fuente: Elaboración propia con base en el Copeems (2012)

Entonces, ya sea que los estudiantes de EMS le tengan confianza o no a directores, maestros orientadores, padres o amigos, o bien reciban alguna influencia negativa de los padres, familiares, parejas o amigos, esto no afectará el rendimiento académico que genera la deserción escolar en EMS. Este tipo de rendimiento está dado por otros factores. 


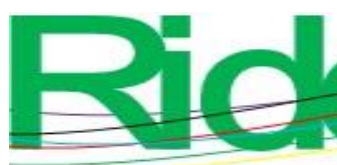

Revista Iberoamericana para la

Investigación y el Desarrollo Educativo

ISSN $2007-7467$

\section{Discusión}

Con base en la información contenida en la tabla 1, se aprecia que el nivel de confianza hacia directores, maestros, orientadores, padres, amigos o hermanos y la influencia negativa que pudieran haber ejercido padres, familiares, parejas o amigos para abandonar los estudios no está relacionado con el bajo rendimiento académico que lleva a la deserción escolar en EMS.

Si bien, parte de la teoría expuesta en la presente investigación relaciona las construcciones Confianza e Influencia negativa con Rendimiento académico, estas parecen generar solo alguna tendencia y no ser determinantes. Más bien son otros factores los que influyen en el rendimiento académico que a la postre llevan a la deserción escolar. Así lo refieren Sánchez et al. (2017) y Oviedo y Oviedo (2017), quienes si bien afirman que el rendimiento académico está relacionado con la atención de los padres hacia las actividades escolares de sus hijos y a la armonía del entorno familiar, le dan mayor peso a factores motivacionales, las tácticas de estudio empleadas de los propios alumnos, así como el método de enseñanza empleado por los docentes, quienes, por su parte, más que inspirar confianza o influir en los estudiantes generan ambientes que facilitan el quehacer académico, la colaboración y el autoaprendizaje, además de que evalúan procesos de enseñanza y aprendizaje. A lo anterior, Chimborazo y Zoller (2018) agregan que el aprendizaje que beneficia al rendimiento académico se da con el tiempo, la práctica y la reflexión.

Ahora, por supuesto que es importante que el docente inspire confianza e influya positivamente en el alumno pues este debe propiciar el desarrollo social y afectivo, pero lo principal para evitar la reprobación, el ausentismo e incrementar los promedios es sin duda allanar la construcción del conocimiento (Escobar, 2015); y en esa línea destaca el grado de responsabilidad que se tenga con la docencia, lo competente que sea en las unidades de aprendizaje que imparta, compartir experiencias, clarificar dudas, construir espacios para la participación grupal e instruir de manera clara (Covarrubias y Piña, 2004). Lemus et al. (2015) relacionaron el rendimiento académico con el grado en que los maestros son capaces de clarificar los procesos, ejercer evaluaciones congruentes con el aprendizaje, elaborar actividades y exámenes y el grado en que son capaces de motivar la autonomía estudiantil. Torres et al. (2014) apreciaron que más que inspirar confianza e influir en el estudiante, la planta docente debe implicar a los estudiantes en su propio aprendizaje. Alcántara (2009), a su vez, afirma que el trabajo de los profesores es planificar sus contenidos, desarrollar trabajos en clase y tareas y dar retroalimentación, además de identificar las áreas de oportunidad y estimular el desarrollo de sus fortalezas. Barragán et al. (2009) informaron que el 


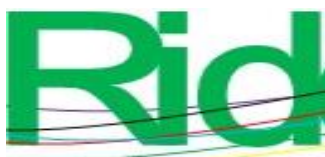

Revista Iberoamericana para la

Investigación y el Desarrollo Educativo

ISSN $2007-7467$

rendimiento académico se encuentra en función directa con el quehacer docente, como organizar y seriar los planes y programas de estudio, desarrollarlos con precisión y lanzar cuestionamientos al grupo. Finalmente, Cuéllar (2017) agregó a las cualidades aquí mencionadas la destreza para retener la atención de los alumnos.

Por otro lado, Campos et al. (2010) se orientan a que el rol del estudiante supone un cambio hacia un papel protagónico donde se hace responsable de su propio avance, por lo que debe tonificar habilidades de gestión y saber trabajar en equipo, más allá de depender de factores que no están bajo su control. Fonseca (2016) encontró que la deserción escolar manifestada por el bajo rendimiento académico se daba por problemas de apatía motivada, por no hallar sentido al estudio ni a lo visto en clase, así como a la vocación institucional que le da a la EMS, los que se pudieran considerar más bien factores personales, independientemente de la confianza o las influencias negativas de familiares o docentes.

En cuanto a los directivos, los resultados del presente estudio se alinean con Reyes et al. (2017), quienes manifestaron que más que generar confianza e influir en los estudiantes para que tengan un buen rendimiento académico, los directores deben ejercer un liderazgo que asegure la continuidad del aprendizaje y desarrollo y adaptación de los entornos cambiantes.

Ahora bien, Miranda (2018) ubicó que el rendimiento académico depende de las cualidades de la educación brindada por los bachilleratos, así como la facilidad de acceso y la pertinencia según los entornos económicos y sociales. Agrega, asimismo, la calidad de la infraestructura, el mobiliario y equipo, las clases impartidas por los profesores y los materiales didácticos, así como la capacidad de gestión de los directores. Y también identificó que la mayoría de los abandonos se dieron de manera voluntaria, puesto que no lograron adaptarse al entorno de estudio ni a la nueva dinámica social, los cuales, probablemente, inician su manifestación con ausentismos, reprobación y promedios bajos. Factores como la influencia negativa y la confianza de directivos, maestros, padres, hermanos o amigos no fueron identificados.

La SEP (2008) ubica cinco causas que afectan el rendimiento académico: 1) el rezago de lo aprendido con el que concluyen la secundaria, 2) la exclusión de los pobres, 3) alumnos provenientes de zonas rurales e indígenas, 4) la oferta educativa y 5) los beneficios que le ven a la continuación de los estudios. Igualmente, establece que para incrementar el rendimiento académico se deben fortalecer los hábitos y técnicas de estudio. 


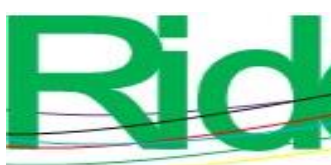

Revista Iberoamericana para la

Investigación y el Desarrollo Educativo

ISSN $2007-7467$

En sus estudios, Tapia et al. (1994) hicieron énfasis en los ambientes económicos y sociales en las que se desenvuelven, en las dinámicas de enseñanza-aprendizaje, las proyecciones que los estudiantes tienen de su propio futuro, el valor que le asignan al bachillerato, los promedios obtenidos en la educación básica y las herramientas y técnicas de enseñanza que emplean los maestros como variables que afectan el rendimiento académico. En dicho estudio, $99 \%$ de los alumnos encuestados consideraron que si se lo propusieran mejorarían su rendimiento académico por sí mismos. Arroyo et al. (2017) y Vidales (2009) identificaron la pasividad de los estudiantes, la deficiencia en los hábitos de estudio, no tomar apuntes en clase, destinar poco tiempo a la instrucción académica, no cumplir con el quehacer escolar e insuficientes ilustraciones docentes como factores que afectan el rendimiento académico.

Los resultados también concuerdan con los obtenidos por Corona, Reyes, Martínez y Rivas (2016), quienes ubicaron que los problemas del rendimiento académico, específicamente la reprobación, se da por la apatía de los alumnos, insuficientes conocimientos de grados previos, maestros que no se actualizan en las técnicas de enseñanza y la falta de infraestructura.

Si bien Martínez et al. (2013) y Barrales et al. (2015) consideran que dentro de los principales causas que afectan el rendimiento académico se encuentran las expectativas familiares, también incluyen factores personales, por ejemplo, la frustración, baja autoestima, depresión y factores educativos como la duración prolongada de los programas de estudio, prácticas pedagógicas deficientes, hábitos incorrectos de estudio, desinterés, insuficiencia de conocimientos o habilidades previas, y expectativas desalentadoras por el desempleo; en sus estudios solo $9.9 \%$ de la reprobación se debió a conflictos familiares, mientras que más de $90 \%$ fue por motivos que incluían la comprensión de asignaturas (63.9 \%) o la inasistencia (11.7\%). Mirete et al. (2015) agregan que 23.8 $\%$ lo hicieron por desinterés. Abril et al. (2008) mencionan la flojera, la poca dedicación al estudio y problemas de comprensión como causantes de bajos rendimientos académicos.

Por su parte, Saucedo et al. (2014) identificaron que $38 \%$ de los estudiantes obtuvieron un bajo rendimiento académico por no entender las explicaciones del profesor, $29 \%$ por no tener interés en la materia, $9 \%$ por la metodología inadecuada del profesor y solo $2 \%$ por tener una mala relación con el profesor. No se mencionó la influencia negativa ni los niveles de confianza salvo, probablemente, la mencionada deficiente relación con el docente.

Finalmente, Poy (2010) informó que en el rendimiento influye la satisfacción que los estudiantes manifiestan hacia sus estudios. Ni la confianza ni la influencia negativa fueron señalados. 


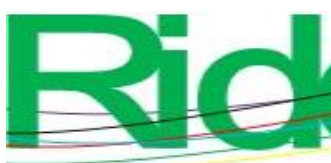

\section{Revista Iberoamericana para la Investigación y el Desarrollo Educativo ISSN $2007-7467$}

Dentro de las fortalezas que se pueden mencionar en el presente estudio se encuentran la amplitud y los recursos empleados, tanto económicos como humanos, así como el tiempo para levantar datos. La encuesta fue aplicada en todo el país a alumnos identificados como desertores, por lo que se puede afirmar que los participantes fueron individuos que abandonaron la EMS, con lo que se le dio confianza y fortaleza a la información. En el otro extremo, se empleó una base de datos del 2012: información más actualizada hubiera sido de mayor conveniencia. Además, a juicio de los autores, el instrumento empleado no abordó de manera profunda conceptos como la repetición y repitencia, los cuales hubieran sido de mucho interés asociarlos con la deserción escolar en futuros estudios.

\section{Conclusiones}

El presente estudio responde a la pregunta de investigación y logra el objetivo trazado. Los resultados muestran que los factores de confianza e influencia negativa no se asocian con el rendimiento académico que lleva a la deserción escolar en EMS.

La confianza que un estudiante le tiene al director, maestros, orientador, papá, mamá, a algún amigo o a sus hermanos para recurrir a ellos cuando tienen problemas en la escuela no se asocia con el rendimiento académico que lleva a la deserción escolar.

En cuanto a la influencia negativa que ejercen la mamá, el papá, familiares, la pareja o amigos para dejar los estudios tampoco se asocia con el rendimiento académico que lleva a la deserción escolar.

Algunos retos en el análisis de los resultados fueron los múltiples factores que intervienen tanto en el rendimiento académico como en la deserción escolar según la teoría analizada. Una dificultad extra, y que nos llevará a futuras investigaciones, es asociar el papel que juega la actitud de los propios alumnos hacia el estudio y su rendimiento académico, así como la predisposición para desertar, pues pareciera que factores internos tienen más peso en el rendimiento académico que elementos externos, como las influencias negativas y la confianza.

Finalmente, algunas recomendaciones para incrementar el rendimiento académico y disminuir las posibilidades de deserción escolar en EMS pudieran ser motivar a los alumnos para que se involucren en su propio aprendizaje mediante acciones específicas como tomar notas de las exposiciones del maestro, programar tiempo para estudiar, fortalecer los hábitos, hacer conciencia de la importancia de entregar deberes. Asimismo, impulsar los grupos de estudio, asesorías grupales, 


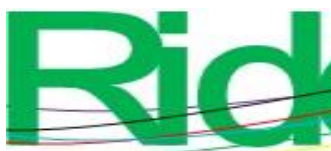

Revista Iberoamericana para la

Investigación y el Desarrollo Educativo

ISSN $2007-7467$

centros complementarios de aprendizaje, tutorías y ayudas psicológicas; capacitar a los estudiantes para aplicar diversas técnicas de estudio, formar círculos de lectura; emprender cursos de inducción a la preparatoria; implementar aulas permanentes donde se consolide lo aprendido en horas de clase, pero con mayor acompañamiento y más aplicación de actividades, incluso revisando contenidos de niveles inferiores o conceptos alternativos pero relacionados con la materia.

También es importante hacer un llamado a los padres de familia para que participen en redes de apoyo académico para sus hijos; e independientemente del resultado de este estudio, promover actividades que generen mayor confianza entre maestros y alumnos y que tanto padres de familia como maestros proyecten metas elevadas en áreas de asistencia, promedios y aprobaciones de materias; programar cursos de aceleración del aprendizaje o que faciliten la transición del nivel básico al medio superior, implementar políticas públicas que garanticen la permanencia escolar como suministro de alimentos y campañas de salud; impulsar el manejo de habilidades socioemocionales; establecer compromisos de los alumnos; darle valor a la educación no formal, disminuir la carga académica y aumentar los niveles educativos obligatorios; generar comunidades dialogantes, con espacios comunes para la reflexión; promover el cambio de actitudes y valores, que la educación sea continua y privilegiar el autoaprendizaje; guiar la toma de decisiones para la vida, fortalecer las competencias comunicativas, matemáticas y lógicas, promocionar la tecnología en casa para elaborar tareas; reconocer los buenos promedios, las materias aprobadas y los niveles de asistencia; fortalecer la identidad y el agrado de los jóvenes por la escuela y estimular la búsqueda de ayuda cada vez que se tenga problemas con las unidades de aprendizaje, poner en práctica los apoyos entre iguales y coadyuvar en la elaboración de planes de carrera y planes de vida. 


\section{Referencias}

Abril, E., Román, R., Cubillas, M. y Moreno, I. (2008). ¿Deserción o autoexclusión? Un análisis de las causas de abandono escolar en estudiantes de educación media superior en Sonora, México. Revista Electrónica de Investigación Educativa, 10(1), 1-16.

Alcántara, L. (2009). Profesores autorregulados. Revista Mexicana de Investigación Educativa, 14(43), 1219-1248.

Alegre, M. y Pérez. R. (2010). Los factores del abandono educativo temprano. España en el marco europeo. Revista de Educación, (número extraordinario), 65-92.

Antelm, A., Gil, A y Cacheiro, M. (2015). Análisis del fracaso escolar desde la perspectiva del alumnado y su relación con el estilo de aprendizaje. Revista Educación y Educadores, 18(3), 471-489.

Arroyo, E., Montes, A., Reyes, O., Zamudio, V. y Tapia, D. (2017). La deserción escolar en la educación media superior en el COBAEH plantel Tula. Xikua. Boletín Científico de la Escuela Superior de Tlahuelilpan, 5(10). Recuperado de http://www.uach. edu.mx/seige/boletín/tlahuelilpan/.

Artavia, J. (2005). Actitudes de los docentes hacia el apoyo académico que requieren los estudiantes con necesidades educativas específicas. Revista Pensamiento Actual, 5(6), 61-70.

Barragán, A., Aguilar, M., Cerpa, G. y Núñez, H. (2009) Relaciones docente-alumno y rendimiento académico. Un caso del Centro Universitario de Ciencias Exactas e Ingenierías de la Universidad de Guadalajara. Sinética. Revista Electrónica de Educación. 33, 1-15.

Barrales, A., Gómez, A. y Guerrero, L. (2015). Factores que originan la reprobación en los estudiantes de bachillerato: caso Colegio Motolinía. En Hernanz, J. A. y Watty, M. L. (coords.), Tendencias y desafíos en la innovación educativa: un debate abierto (pp. 881-890). Veracruz, México: Universidad Veracruzana.

Camacho, M. (2018). Fracaso escolar y abandono educativo temprano en Huelva. Prácticas en positivo y propuestas de mejora. Sevilla, España: Universidad Internacional de Andalucía.

Campos, J., Brenes, O. y Solano, A. (2010). Competencias del docente de educación superior en línea. Revista Electrónica Actualidades Investigativas en Educación, 10(3), 1-19.

Casquero, A. y Navarro, M. (2010). Determinantes del abandono escolar temprano en España: un análisis por género. Revista de Educación, (número extraordinario), 191-223. 
Cernadas, A. y Pérez, M. (2014). Un análisis del fracaso escolar en dos centros de educación secundaria. Revista de Estudios e Investigación en Psicología y Educación, 1(2), 122-131.

Cimborazo, C. y Zoller, M. (2018). Condiciones de la innovación educativa. Revista Dilemas Contemporáneos: Educación, Política y Valores, 6(13), 1-17.

Consejería de Educación de la Junta de Castilla y León [CE]. (2013). Abandono escolar temprano. Factores de abandono y factores de continuidad. Intervenciones posibles desde los centros educativos. España: Consejería de Educación de la Junta de Castilla y León. Recuperado de www.educa.jcyl.es/crol/es/cap acitacion-docente/abandono-esco lar-temprano-factoresabandono-factores-contin.

Consejo para la Evaluación de la Educación del Tipo Medio Superior [Copeems]. (2012). Reporte de la Encuesta Nacional de Deserción en la Educación Media Superior. México: Secretaría de Educación Pública.

Corona, V., Reyes, S., Martínez, S. y Rivas, C. (2016). Estrategias para la disminución de los índices de reprobación en el Instituto Tecnológico de Pachuca. Revista de sistemas y Gestión Educativa, 3(9), 62-69.

Covarrubias, P. y Piña, M. (2004). La interacción maestro-alumno y su relación con el aprendizaje. Revista Latinoamericana de Estudios Educativos, 34(1), 47-84.

Cuéllar, D. (2017). Abandono escolar en educación media superior. Análisis de factores escolares asociados a altas tasas de abandono. Ponencia presentada en el Congreso Nacional de Investigación Educativa. México.

Diario Oficial de la Federación [DOF]. (2008). Acuerdo 447 por el que se establecen las competencias docentes para quienes imparten educación media superior en las modalidades no escolarizada y mixta. Diario Oficial de la Federación. Recuperado de http://www.sems. gob.mx/work/models/sems/Resource/11435/1/images/5_4_acuerdo_447_competencias_do centes_ems.pdf.

Díaz, K. y Osuna, C. (2017). Contexto sociofamiliar en jóvenes en situación de abandono escolar en educación media superior. Perfiles Educativos, 39(158), 70-91.

Dzay, F. y Narváez, Ó. (2012). La deserción escolar desde la perspectiva estudiantil. Quintana Roo, México: Universidad de Quintana Roo.

El Sahili, L. (2011). Informe de investigación sobre deserción escolar mediante jerarquización de factores en la UPIIG del IPN en Silao, Guanajuato. REXE. Revista de Estudios y Experiencias en Educación, 10(19), 33-48. 


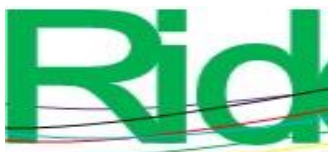

Escobar, M. (2015). Influencia de la interacción alumno-docente en el proceso enseñanzaaprendizaje. Paakat: Revista de Tecnología y Sociedad, 5(8), 1-9.

Espínola, V. y Claro, J. (2010). Estrategias de prevención en la educación secundaria: perspectiva latinoamericana. Revista de Educación, (número extraordinario), 257-280.

Fernández, J. (2012). Competencias docentes y educación inclusiva. Revista Electrónica de Investigación Educativa, 15(2), 82-99.

García, E., García, A. y Reyes, J. (2014). Relación maestro alumnos y sus implicaciones con el aprendizaje. Ra-Ximhai, 10(5), 279-290.

George, D. and Mallery, P. (2003). SPSS for Windows step by step: A simple guide and reference $\left(4^{\text {th }}\right.$ ed.). Boston, United States.

Gómez, M. y Vázquez, E. (2014). Deserción a nivel medio superior en escuela superior Tepeji. Tepexi. Boletín Científico de la Escuela Superior Tepeji, 1(2), 1-5.

González, D. (2002). Las dificultades de aprendizaje en el aula. España: Ediciones edebé.

Infante, R. y Parra, L. (2010). Deserción escolar y desarrollo social: Una mirada sobre el programa Volver a la Escuela en Bogotá. Revista Educación y Desarrollo Social, 4(2), 75-86.

Instituto Nacional para la Evaluación de la Educación [INEE]. (2017). Directrices para mejorar la permanencia escolar en la educación media superior. México: Instituto Nacional para la Evaluación de la Educación. Recuperado de www.inee.edu.mx.

Juárez, É. (2018). Abandono escolar en bachillerato: una mirada a las raíces del fenómeno. Red, 11, 1-12. Recuperado de www.inee.edu.mx/index.php/655/blog-revista-red-11-2018.

Kopyto, I. (2017). La relación entre el docente y el estudiante. Escritos en la Facultad, 13(136), 2223.

Landeros, J. (2012). Deserción en la educación media superior en México. México: Suma X la Educación.. Recuperado de: editor.pbsiar.com/upload/PDF/desercion/pdf.

Lara, B., González, A. González, M. y Martínez, M. (2017) Fracaso escolar: conceptualización y perspectivas de estudio. Revista de Educación y Desarrollo, 30, 71-83.

Lemus, L., Torres, F., Serrano, M. y Guzmán, G. (2015). La tutoría: eje fundamental para mejorar el rendimiento académico de alumnos de nivel superior. Innovación Educativa. 15(67), 89-110

López, E., Velázquez, J. y Ibarra, G. (2011). Causas de la deserción escolar de nivel medio superior en Baja California. Ponencia presentada en el XI Congreso Nacional de Investigación Educativa. México. 


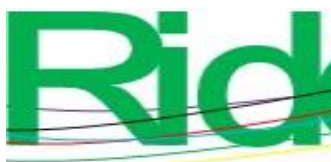

Revista Iberoamericana para la

Investigación y el Desarrollo Educativo

ISSN $2007-7467$

Martínez, V. (2009). Investigación y reflexión sobre condicionantes del fracaso escolar. Revista Latinoamericana de Estudios Educativos, 39(1 y 2), 11-38.

Martínez, A., Hernández, L., Carrillo, D., Romualdo, Z. y Hernández, C. (2013). Factores asociados a la reprobación estudiantil en la Universidad de la Sierra Sur, Oaxaca. Temas de Ciencia y Tecnología, 17(51), 25-33.

Mena, L., Fernández, M. y Riviére, J. (2010). Desenganchados de la educación: procesos, experiencias, motivaciones y estrategias del abandono y del fracaso escolar. Revista de Educación, (número extraordinario), 119-145.

Ministerio de Educación, Cultura y Deporte [MECD]. (2012). Educación inclusiva. Iguales en la diversidad. España: Ministerio de Educación, Cultura y Deporte. Recuperado de http://www.ite.educacion.es/ formacion/ materiales/126/cd/.

Miranda, F. (2018). Abandono escolar en educación media superior: conocimiento y aportaciones de política pública. Sinética. Revista Electrónica de Educación, 51, 1-22.

Mirete, A., Soro, M. y Maquilón, J. (2015). El fracaso escolar y los enfoques de aprendizaje: medidas para la inclusión educativa. Revista Electrónica Interuniversitaria de Formación del Profesorado, 18(3), 183-196.

Mora, A. (2010). Determinantes del abandono escolar en Cataluña: más allá del nivel socioeconómico de las familias. Revista de Educación, (número extraordinario), 171-190.

Moreno, B., Sandoval, M. y Valdés, R. (2015). Las concepciones de los docentes sobre la tutoría. Un estudio en el nivel medio superior de la Universidad Autónoma del Estado de México. Revista Dilemas Contemporáneos: Educación, Política y Valores, 2(3), 1-28.

Moreno, D. (2013). La deserción escolar: un problema de carácter social. In Vestigium Ire, 6, 115124.

Muñiz, C., Maldonado, L., Leyva, O., López, R., Saldierna, A., Hernández, T. y Rodríguez, E. (2011). Hábitos comunicativos y sofisticación política. Comunicación, Política y Ciudadanía, 1, 237-253.

Organista, J. (2010). Análisis del uso de objetos de aprendizaje en las materias de matemáticas y física de bachillerato. Sinética. Revista Electrónica de Educación, 34, 1-16.

Ortega, P., Macías, M. y Hernández, M. (2014). Causas de la deserción escolar en las telesecundarias de la zona 55. Revista Xihmai, 8, 31-54.

Oviedo, J. y Oviedo, E. (2017). Culturas de profesores y reformas educativas. Revista Iberoamericana para la Investigación y el Desarrollo Educativo, 7(14), 1-29. 


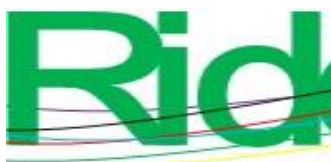

\section{Revista Iberoamericana para la \\ Investigación y el Desarrollo Educativo \\ ISSN $2007-7467$}

Piña, J., Escalante, A., Ibarra, L. y Fonseca, C. (2017). El modelo basado en competencias. Representaciones sociales de docentes de educación media superior. Nueva Época, 10(41), 158-178.

Ponce, V. (2004). Reprobación y fracaso escolar en secundaria. Hacia una reforma integral. Revista de Educación y Desarrollo, 2, 59-70.

Poy, R. (2010). Efectos del credencialismo y las expectativas sociales sobre el abandono escolar. Revista de Educación, (número extraordinario), 147-169.

Reyes, V., Trejo, M. y Topete, C. (2017). El liderazgo directivo y la gestión en el nivel medio superior del Instituto Politécnico Nacional de México: una mirada desde los estudiantes. Revista Iberoamericana para la Investigación y el Desarrollo Educativo, 8(15), 1-35.

Roca, E. (2009). El abandono temprano de la educación y la formación en España. Revista de Educación, (número extraordinario), 31-62.

Román, M. (2013). Factores asociados al abandono y la deserción escolar en América Latina: Una mirada en conjunto. Revista Iberoamericana sobre Calidad, Eficacia y Cambio en la Educación, 11(2), 33-59.

Romero, E. y Hernández, M. (2019). Análisis de las causas endógenas y exógenas del abandono escolar temprano: una investigación cualitativa. Educación XX1, 22(1), 263-293.

Rossado, V. (2016). Enseñanza en la era digital: La empatía docente y el aprendizaje colaborativo. Ponencia presentada en el XX Congress of Iberoamerican Society of Digital Graphics. Buenos Aires, del 9 al 11 de noviembre de 2016. Recuperado de https://www.researchgate.net/publication/311610030_Ensenanza_en_la_Era_Digital_La_E mpatia_Docente_y_el_Aprendizaje_Colaborativo.

Ruiz, R., García, J. y Pérez, M. (2014). Causas y consecuencias de la deserción escolar en el bachillerato: Caso Universidad Autónoma de Sinaloa. Ra Ximhai, 10(5), 51-74.

Salvà, F., Oliver, M. y Comas, R. (2014). Abandono escolar y desvinculación de la escuela: perspectiva del alumnado. Revista Internacional de Investigación en Educación, 6(13), 129142.

Sánchez, J. y Gaya, J. (2014). La empatía en la docencia. Recuperado de https://educacionadistancia.juntadeandalucia.es/profesorado/autoformacion/pluginfile.php/ 3144/mod_book/chapter/2168/La_empat\%C3\%ADa_en_la_docencia.pdf. 
Sánchez, M., Téllez, M., Sánchez, J. y Reyes, V. (2017). Conceptualizando las necesidades de actualización y profesionalización docente en el nivel medio superior. Revista Dilemas Contemporáneos: Educación, Política y Valores, 4(3), 1-33.

Saucedo, M., Herrera, S., Díaz, J., Bautista, S. y Salinas, H. (2014). Indicadores de reprobación: Facultad de Ciencias Educativas. RIDE Revista Iberoamericana para la Investigación y el Desarrollo Educativo, 5(9), 1-12.

Silvera, L. (2016). La evaluación y su incidencia en la deserción escolar: ¿falla de un sistema, de las instituciones educativas, del docente o del estudiante? Revista Educación y Humanismo, $18(31), 313-325$.

Secretaría de Educación Pública [SEP]. (2014a). Movimiento contra el abandono escolar en la educación media superior. México: Secretaría de Educación Pública. Recuperado de www.sems.gob.mx/work/ models/sems /recursos/11390/ images/10_Movimiento_contra_ Abandono_Escolar_Caja_de_Herramientas.pdf.

Secretaría de Educación Pública [SEP]. (2014b). Caja de herramientas para la gestión contra el abandono escolar. México: Secretaría de Educación Pública. Recuperado de www.sems.gob.mx/work/models/sems /recursos/11390/images/ 10_Movimiento_contra_Abandono_Escolar_Caja_de_Herramientas.pdf.

Secretaría de Educación Pública [SEP]. (2015). Análisis del movimiento contra el abandono en la educación media superior. México: Secretaría de Educación Pública.

Sistema Educativo Estatal. (2011). Deserción escolar en el nivel medio superior. Baja California, México: Gobierno del Estado de Baja California.

Tapia, M., Tamez, A. y Tovar, A. (1994). Causas de reprobación en los Colegios de Bachilleres del Estado de Morelos. Revista Latinoamericana de Estudios Educativos, 24(1 y 2), 107-128.

Torres, A., Badillo, M., Valentín, N. y Ramírez, E. (2014). Las competencias docentes: El desafío de la educación superior. Innovación Educativa, 14(66), 129-145.

Venegas, G., Chilusia, M., Castro, S. y Casillas, I. (2017). La deserción en la educación. Boletín Virtual, 6(4), 235-239.

Vera, A. y Mazadiego, T. (2010). Una perspectiva sobre las actitudes y el deber ser de los docentes en el aula escolar. Revista de Educación y Desarrollo, 14, 53-58.

Vidales, S. (2009). El fracaso escolar en la educación media superior: El caso del bachillerato de la Universidad Mexicana. Revista Iberoamericana sobre Calidad, Eficacia y Cambio en Educación, 7(4), 320-341. 
Welch, S. and Comer, J. (1988). Quantitative Methods for Public Administration: Techniques and Applications. United States: Dorsey Press.

\begin{tabular}{|c|c|}
\hline Rol de Contribución & Autor (es) \\
\hline Conceptualización & $\begin{array}{l}\text { David Fernando Lozano Treviño (principal) y Lauro Maldonado Maldonado (que } \\
\text { apoya) }\end{array}$ \\
\hline Metodología & $\begin{array}{l}\text { David Fernando Lozano Treviño (principal) y Lauro Maldonado Maldonado (que } \\
\text { apoya) }\end{array}$ \\
\hline Software & Lauro Maldonado Maldonado \\
\hline Validación & $\begin{array}{l}\text { David Fernando Lozano Treviño (principal) y Lauro Maldonado Maldonado (de } \\
\text { apoyo) }\end{array}$ \\
\hline Análisis Formal & $\begin{array}{l}\text { David Fernando Lozano Treviño (principal) y Lauro Maldonado Maldonado (de } \\
\text { apoyo) }\end{array}$ \\
\hline Investigación & David Fernando Lozano Treviño \\
\hline Recursos & $\begin{array}{l}\text { David Fernando Lozano Treviño (Con apoyo de la Universidad Autónoma de } \\
\text { Nuevo León) }\end{array}$ \\
\hline Curación de datos & David Fernando Lozano Treviño \\
\hline $\begin{array}{l}\text { Escritura - Preparación del } \\
\text { borrador original }\end{array}$ & David Fernando Lozano Treviño \\
\hline Escritura - Revisión y edición & $\begin{array}{l}\text { David Fernando Lozano Treviño (principal) y Lauro Maldonado Maldonado (de } \\
\text { apoyo) }\end{array}$ \\
\hline Visualización & $\begin{array}{l}\text { David Fernando Lozano Treviño (principal) y Lauro Maldonado Maldonado (de } \\
\text { apoyo) }\end{array}$ \\
\hline Supervisión & David Fernando Lozano Treviño \\
\hline Administración de Proyectos & David Fernando Lozano Treviño \\
\hline Adquisición de fondos & $\begin{array}{l}\text { David Fernando Lozano Treviño (principal) y Lauro Maldonado Maldonado (de } \\
\text { apoyo) }\end{array}$ \\
\hline
\end{tabular}

